$=\lambda^{4}-4 \lambda^{3}-73 \lambda^{2}+260 \lambda+568$ in the neighborhood of the stated roots and showed that the error in the eigenvalues was less than $1 \times 10^{-7}$.

The last component of $x^{(4)}$ in [1] should read $-0.38333123+$ in place of $-0.3833123+$.

Computing Laboratory

J. L. BRENNER

G. W. REITWIESNER

Ballistic Research Laboratories

Ordnance Corps, U. S. Army

Aberdeen Proving Ground, Maryland

Sponsored by the Office of Ordnance Research, U. S. Army.

1 E. BODEWIG, "A practical refutation of the iteration method for the algebraic eigenproblem," $M T A C$, v. 8,1954, p. $237-239$.

${ }^{2}$ R. T. GREGORY, "Computing eigenvalues and eigenvectors of a symmetric matrix on the ILLIAC," $M T A C$, v. 7, 1953, p. 215-220.

${ }^{3}$ C. G. J. JACOBI, "Ein leichtes verfahren . . .", Jn. reine angew. Math., v. 30, 1846, p. 51-95.

\title{
A Note on the Summation of Chebysher Series
}

In a recent note [1] I gave tables of the numerical coefficients in the Chebyshev expansions of some common functions, and suggested that these should be used to provide polynomial approximations by truncation and rearrangement in powers of the independent variable.

The purpose of the present note is to show that the truncated Chebyshev series may be evaluated directly without rearrangement, and without using tables of Chebyshev polynomials. The process used is one of recurrence, and is well suited for use with automatic computing machines.

Let the truncated series be denoted by

$$
f(x)=A_{0}+A_{1} T_{1}{ }^{*}(x)+A_{2} T_{2}{ }^{*}(x)+\cdots+A_{N} T_{N}{ }^{*}(x),
$$

where, following the notation of LANCzos [2] which is used throughout,

$$
T_{n}{ }^{*}(x)=\cos n \theta, \quad \cos \theta=2 x-1, \quad 0 \leqslant x \leqslant 1 .
$$

In order to evaluate $f(x)$ for a given value of $x$ we construct the sequence $B_{N}$, $B_{N-1}, \cdots, B_{0}$, given by $B_{N+2}=B_{N+1}=0$ and

$$
B_{n}-(4 x-2) B_{n+1}+B_{n+2}=A_{n}, \quad n=N, N-1, \cdots, 1,0 .
$$

Then we have

$$
f(x)=B_{0}-(2 x-1) B_{1} .
$$

This result may be verified by using the recurrence relation

$$
T_{n}^{*}(x)-(4 x-2) T_{n+1}^{*}(x)+T_{n+2}^{*}(x)=0 .
$$

It may be thought that the coefficient $(4 x-2)$ in $(2)$ can give rise to considerable building-up error in $f(x)$. To investigate this point, we observe that the general solution of the equation

$$
u_{n}-(4 x-2) u_{n+1}+u_{n+2}=0
$$


is given by

$$
u_{n}=\alpha T_{n}^{*}(x)+\beta U_{n}^{*}(x),
$$

where $U_{n}^{*}(x)=\sin (n+1) \theta \operatorname{cosec} \theta, \cos \theta=2 x-1$, and $\alpha, \beta$ are arbitrary constants.

Hence a rounding error $\epsilon(n)$ in $A_{n}$ or $B_{n}$ introduces an error $\epsilon_{8}(n)$ in $B_{s}(s \leqslant n)$, given by

$$
\epsilon_{s}(n)=\alpha T_{s}^{*}(x)+\beta U_{s}^{*}(x),
$$

where

$$
\left.\begin{array}{rl}
\epsilon(n) & =\alpha T_{n}^{*}(x)+\beta U_{n}^{*}(x) \\
0 & =\alpha T^{*}{ }_{n+1}(x)+\beta U^{*}{ }_{n+1}(x)
\end{array}\right\} .
$$

Solving the last pair of equations, we obtain

$$
\alpha=\epsilon(n) \cdot U^{*}{ }_{n+1}(x) / T_{1}{ }^{*}(x), \quad \beta=-\epsilon(n) \cdot T^{*}{ }_{n+1}(x) / T_{1}{ }^{*}(x)
$$

and substituting in (4) we find that

$$
\begin{aligned}
\epsilon_{s}(n) & =\left\{T_{s}^{*}(x) \cdot U^{*}{ }_{n+1}(x)-U_{s}^{*}(x) \cdot T^{*}{ }_{n+1}(x)\right\} \epsilon(n) / T_{1}{ }^{*}(x) \\
& =U^{*}{ }_{n-8}(x) \cdot \epsilon(n) .
\end{aligned}
$$

The corresponding error in $f(x)$ may be found from (3), and is given by

$$
\begin{aligned}
\epsilon_{0}(n)-(2 x-1) \epsilon_{1}(n) & =\left\{U_{n}{ }^{*}(x)-T_{1}^{*}(x) \cdot U^{*}{ }_{n-1}(x)\right\} \epsilon(n) \\
& =T_{n}{ }^{*}(x) \cdot \epsilon(n),
\end{aligned}
$$

which is the same as that produced by an error of $\epsilon(n)$ in $A_{n}$ when the series is summed in the usual way. Hence the rounding-off of $B_{n}$ to the same number of decimals as $A_{n}$ can only double the maximum rounding error in $f(x)$. If one or two guarding figures are retained in $A_{n}$ and $B_{n}$, this error may be made negligible compared with the truncation error which has been introduced in replacing the infinite Chebyshev series by $f(x)$.

Thus, although the errors in $B_{n}$ may become quite large if $N$ is large (see (5)), the error in $f(x)$ is not serious.

We may note that a similar device may be used to evaluate other series in terms of functions which satisfy a linear recurrence relation; for example, Neumann series of Bessel functions.

Let

where

$$
f(x)=a_{0} p_{0}(x)+a_{1} p_{1}(x)+\cdots+a_{N} p_{N}(x),
$$

$$
p_{n+1}(x)+\alpha_{n} p_{n}(x)+\beta_{n} p_{n-1}(x)=0,
$$

and $\alpha_{n}, \beta_{n}$ may be functions of $x$ as well as of $n$.

We construct the sequence $b_{N}, b_{N-1}, \cdots, b_{0}$, where $b_{N+1}=b_{N+2}=0$ and

$$
b_{n}+\alpha_{n} b_{n+1}+\beta_{n+1} b_{n+2}=a_{n}, \quad n=N, N-1, \cdots, 0 .
$$


Then application of the recurrence relation (6) shows that

$$
f(x)=b_{0} p_{0}(x)+b_{1}\left\{p_{1}(x)+\alpha_{0} p_{0}(x)\right\} .
$$

National Physical Laboratory

C. W. Clenshaw

Teddington, Middlesex

England

This note is published with the permission of the Director of the National Physical Laboratory.

$1 \mathrm{C}$. W. Clenshaw, "Polynomial approximations to elementary functions," MTAC, v. 8, 1954, p. 143-147.

${ }_{2}$ NBS Applied Mathematics Series 9, Tables of Chebyshev Polynomials $S_{n}(x)$ and $C_{n}(x)$. U. S. Govt. Printing Office, Washington, 1952.

\section{Conjectures Concerning the Mersenne Numbers}

Conjectures concerning the Mersenne numbers are appropriate since they were inaugurated with one. A conjecture [1] that seems likely to be false, but unlikely to be proved false, is that all numbers $p_{n}$ are prime $(n=1,2,3, \cdots)$, where, for example, $p_{4}$ is

$$
2^{2^{2}-1}-1
$$

Recursively, $p_{1}=3, p_{n+1}=2^{p_{n}}-1$. The first four are $3,7,127$ and $2^{127}-1$, all known to be prime. Any factor of $p_{5}$ is congruent to 1 modulo $p_{4}$, so $p_{5}$ certainly has no factor less than $2^{127}$. Similarly

$$
2^{2^{2281}-1}-1
$$

is not divisible by any known prime, if $2^{2281}-1$ is still the largest known prime [2]. One can try to argue about the probability that a number of the form $2^{p}-1$ is prime, when $p$ is known to be prime. The probability that a whole number $x$ is prime is about $1 / \log x$, and is close to

$$
\frac{1}{2} e^{\gamma}\left(1-\frac{1}{2}\right)\left(1-\frac{1}{3}\right)\left(1-\frac{1}{5}\right) \cdots\left(1-\frac{1}{q}\right)
$$

where $q \doteqdot \sqrt{x}$, so the factors $\left(1-\frac{1}{2}\right)$, $\left(1-\frac{1}{3}\right)$, etc., can be regarded as probabilities that are not far from independent. But if $x=2^{p}-1$, only every $p$ th factor of (1) should be taken, and the probability apparently ought to be about the $p$ th root of $1 / p \cdot \log 2$, which is approximately 1 when $p$ is large. But this argument is also invalid, as we may see from the statistics of Mersenne primes [2]. We may see from these statistics (assuming them to contain no gaps), that, if $m_{n}$ denotes the $n$th Mersenne prime $\left(m_{1}=3\right)$, then

$$
2.18 \log \log m_{n}<n<2.72 \log \log m_{n} \quad(3 \leqslant n \leqslant 17)
$$

while 